\title{
Validation of the Gujarati and Hindi Versions of Modified Falls Efficacy Scale
}

\author{
Joshi $\mathrm{S}^{1 *}$ and Kumar GP2 \\ ${ }^{1}$ Ashok and Rita Patel Institute of Physiotherapy, Charusat University, India \\ ${ }^{2}$ Sumandeep Vidyapeeth, India
}

*Corresponding author: Shuchi Joshi, Assistant Professor, Ashok and Rita Patel

\section{Research Article \\ Volume 1 Issue 1}

Received Date: September 18, 2018

Published Date: October 25, 2018

DOI: $10.23880 /$ aphot- 16000106

Institute of Physiotherapy, Charusat university, India, Tel: 91 9714753839; Email: shuchijoshi.phy@charusat.ac.in

\section{Abstract}

Background: Frail, old patients with and without cognitive impairment are at high risk of falls and associated medical and psychosocial issues. The Modified Falls Efficacy Scale (MFES) was developed to assess fear of falling and it is shown to be a reliable and valid measure of falls self-efficacy in western countries. The lack of adequate, validated instruments has partly hindered research in this field.

Methodology: The 'forward-backward' translation procedure was applied to translate the MFES into Hindi and Gujarati. Harmonization was done by expert panel review and pilot testing over 10 subjects. The questionnaires were then finalized.98 participants (70-Gujarati MFES; 28-Hindi MFES), meeting inclusion-exclusion criteria and willing to participate were included in the study. Interview regarding general health and fall history was taken. Gujarati MFES and Hindi MFES data were collected and Berg Balance Scale was performed.

Results: The analysis of internal validity of the Gujarati and Hindi MFES revealed that the scale items presented adequate internal consistency (Cronbach's alpha: Gujarati MFES- 0.993, Hindi MFES-0.992).The Gujarati MFES showed strong positive correlation with BBS $(r=0.820, P$ value $=0.000)$ and a strong negative correlation with age $(r=-0.351$, $P$ value=0.003). The Hindi MFES showed a moderate positive correlation with BBS ( $\mathrm{r}=0.565, \mathrm{P}$ value=0.002) and a weak negative correlation with age $(\mathrm{r}=-0.297, \mathrm{P}$ value $=0.125)$.

Conclusion: The Gujarati version of MFES is a valid and measure to estimate fall-related efficacy in older adults who have Gujarati as their first language. The Hindi version of MFES shall have its validity established with a larger sample size.

Keywords: Fall-Related Efficacy; Validity; Modified Falls Efficacy Scale; Berg Balance Score

\section{Introduction}

Over the next several decades, the elderly people will represent a large segment of the population. In India, a 'senior citizen' or 'older adult' is defined as a person aged
60 years and older [1]. According to WHO, the size of the elderly population in India increased from 20 million in 1951 to 57 million in 1991 and was about 107 million in 2010, which is expected to grow upto 198 million in 2030 and 326 million in 2050 [2]. The average remaining 
length of life is around 18 years (16.7 years for men, 18.9 years for women) at age 60 years and 12 years (10.9 years for men and 12.4 years for women) at age 70 years [1]. The rapid increase in the number of old people in the population also raises various social, economic and health issues. Elderly people have common problems like impaired mobility, falls, impaired cognition, urinary incontinence, etc. Out of these, falls are common events in the lives of older people and can result in a range of adverse outcomes, from minor bruises to fractures, disability, dependence and death [2]. In the US, $30 \%$ of individuals aged 65 years and older fall at least once a year. In Japan, the prevalence of falls was $13.7 \%$, and in China it was $26.4 \%$. In India, the prevalence of falls among older adults aged 60 years and older was $14 \%$ to 53\% [1].

In 1987, the Kellogg International Working Group on the prevention of falls in the elderly defined the falls as unintentionally coming to the ground or some lower level and other than as a consequence of sustaining a violent blow, loss of consciousness, sudden onset of paralysis as in stroke or an epileptic seizure [3]. In other words, a "fall" is when a sudden, unintended loss of balance leaves the individual in contact with the floor or another surface such as a step or chair [2]. A near faller is defined as a person who tends to fall unintentionally but not due to extrinsic factor. He /she are supported by somebody else nearby in the event, otherwise he/she would suffer from fall.

Falls can result from diverse causes which can be classified into intrinsic and extrinsic factors. Intrinsic factors commonly include postural hypotension, weak lower limb strength, impaired balance and slow gait speed. Extrinsic factors include uneven ground, wet floor or dark environment. Falls and fear of falling is closely correlated [4].

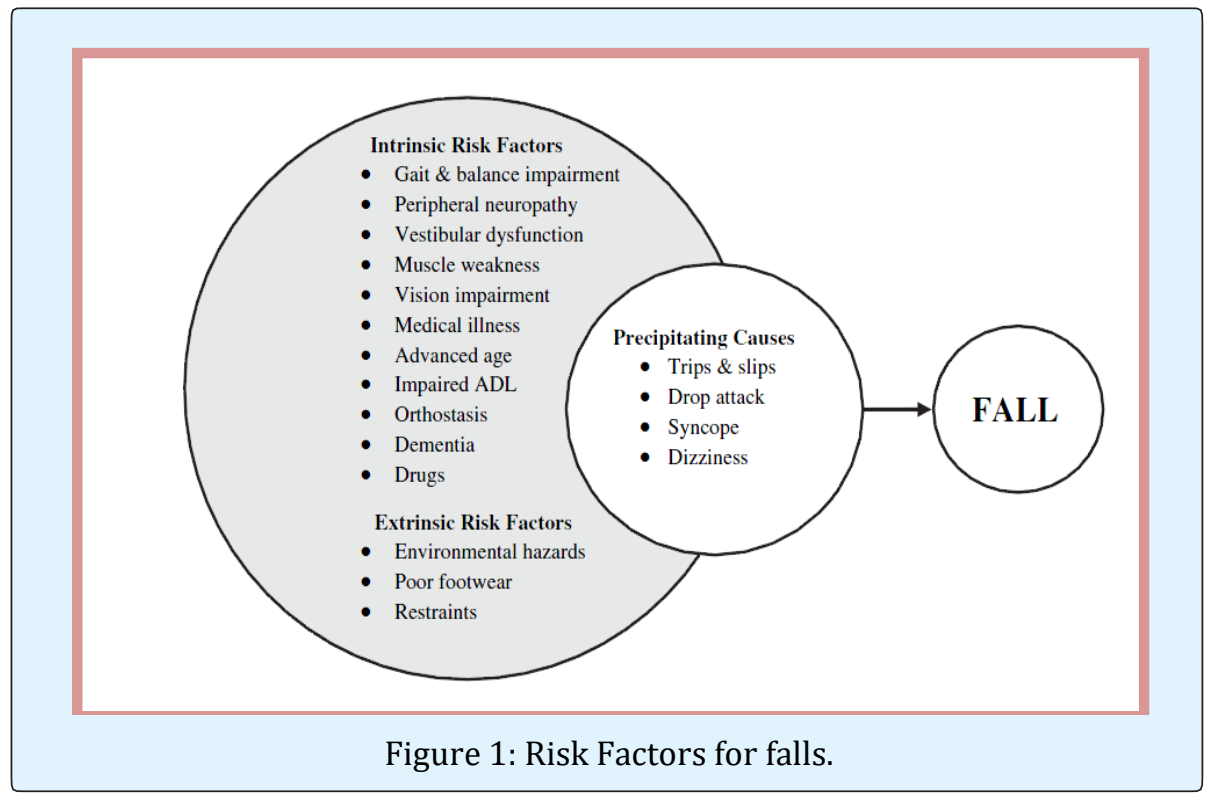

Evidences suggest that fall-related injuries in older adults are age and gender related, leading to high healthcare consumption, costs, and long-term reduced quality of life. Further implementation of falls prevention strategies is needed to control the burden of fall-related injuries in the aging population. They can result in a range of adverse outcomes, from minor bruises to fractures, disability, dependence and death [5]. In 2008, 64\% of adults over 65 years of age reported limitations in at least one domain of physical function-walking, climbing, standing, sitting, stooping, reaching, grasping, carrying, and/or pushing [6].
Older people who have suffered a fall are at increased risk of falling again. In a prospective study of 325 community dwelling persons who had fallen in the previous year, Nevitt, et al. found that 57 experienced at least one fall in a 12 -month follow up period and $31 \% \mathrm{had}$ 2 or more falls. Falling is also more prevalent in frailer older people than vigorous ones, in those who had difficulties undertaking ADLs, and in those with particular medical conditions that affect posture, balance and gait. In independent older community dwelling people, about $50 \%$ of falls occur within their homes and immediate surroundings. Most falls occur on level surfaces within community used rooms such as bedroom, living room and 
kitchen. Comparatively few falls occur in the bathroom, on stairs or from ladders and stools. The remaining falls occur in public places and other people's homes.

The location of falls is related to age, sex and frailty. In community dwelling elderly women, the number of falls occurring outside the home decreased with age, with a corresponding increase in the number of falls occurring inside the home on a level surface. Campbell et al. found that fewer men than women fell inside the home $(44 \%$ versus $65 \%)$ and more men fell outside $(25 \%$ versus $11 \%)$. Thus the occurrence of falls is strongly related to exposure, i.e. they occur in situations where older people are undertaking their usual daily activities. Most falls occur during periods of maximum activity in the morning or afternoon, and only about $20 \%$ occur between 9 p.m. and 7 a.m.

Depending on the population under study, between $22 \%$ and $60 \%$ of older people suffer minor injuries from falls, $10-15 \%$ suffers serious injuries, $2-6 \%$ suffers fractures and $0.2-1.5 \%$ suffers hip fractures. The most commonly self-reported injuries include superficial cuts and abrasions, bruises and sprains. The most common injuries that require hospitalization comprise femoral neck fractures, other fractures of the leg, radius, ulna and other bones in the arm and fractures of the neck and trunk. Elderly people recover slowly from hip fractures and are vulnerable to post-operative complications. In many cases, hip fractures result in death and those who survive, may never regain complete mobility.

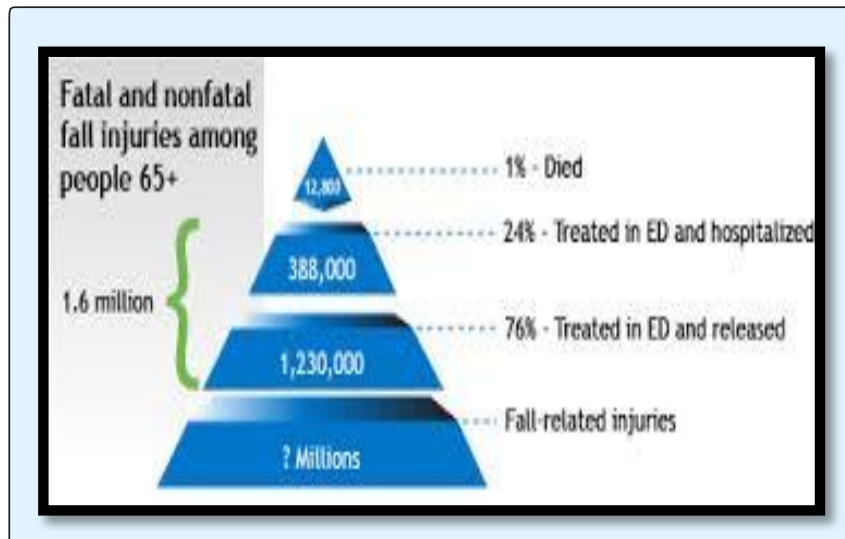

Figure 2: Fall Related Injuries (Source: Centre of Excellence for Falls Prevention).

Community studies that are limited to elderly people who have actually fallen have reported prevalence rates of $83 \%$, but strikingly $46 \%$ of community-dwelling elders who have not fallen also report fear of falling [2].
Among elderly persons who are afraid of falling, up to $70 \%$ acknowledge avoiding activities because of this fear. In some cases, individuals become housebound as a result of their fear. Activity restriction is, in itself, a risk factor for falls because it can lead to muscle atrophy, deconditioning and poorer balance. Curtailment of activities can also lead to social isolation. Thus, fear of falling can contribute to both functional decline and impaired quality of life.

The objective of conducting a validity analysis is to ensure that the underlying scale measures what it is supposed to measure. Therefore, a scale is valid to the extent that it measures what it is intended to measure. The validity of a translated questionnaire can be examined by content validity, criterion validity and construct validity. Content validity is defined as the extension to which a measurement reflects the specific intended domain of content. Concurrent validity is a measurement of an agreement in terms of responses collected from a translated questionnaire versus the questionnaire regarded as the gold standard. Construct validity looks into the agreement between a theoretical concept and a specific measuring procedure. Construct validity can further be subdivided into convergent validity and discriminate validity. Convergent validity is a general agreement between measures where theoretically they should be related. On the other hand, discriminate validity is a general disagreement between measures where theoretically they should not be related.

The prevalence of fear of falling was various from $12 \%$ to $65 \%$ of community-dwelling elderly aged of 60 years or above. Falls can result in restriction of activity and fear of falling, reduced quality of life and independence. Even falls that do not result in physical injuries can result in the 'post-fall' syndrome; a loss of confidence, hesitancy, tentativeness, with resultant loss of mobility and independence. It has been found that after falling, $48 \%$ of older people report a fear of falling and $25 \%$ report curtailing activities [7].

Several studies have indicated that people who are afraid of falling appear to enter a debilitating spiral of loss of confidence, restriction of physical activities and social participation, physical frailty, falls, and loss of independence. In addition to the adverse consequences of fear of falling for those suffering from it, there are consequences for the public expenditure, because healthcare utilization increases. It is therefore important to reduce fear of falling by reversing the downward spiral by intervening in factors in the spiral, such as increasing physical functioning, or in predictors of those factors, such as improved medication use. 


\section{Annals of Physiotherapy \& Occupational Therapy}

An important conceptual issue is whether 'fear of falling' is a temporary state, closely related to having had a fall, or whether it is a more long-lasting condition which continues long after fall has occurred. The association between fear of falling and decrease of mobility and quality of life observed in previous studies in some other elderly populations confirms that fear of falling could be a long-lasting condition and that there is a need for interventional studies to prevent and limit the consequences of falls in elderly persons, in particular the development of fear of falling [8].

It is widely recognized that older people with balance disorders suffer from multiple impairments, such as multi-sensory loss, weakness, orthopedic constraints and cognitive impairments [9]. For example, an individual with sensory loss in the feet due to neuropathy may compensate by increasing dependence on visual information-a strategy that result in instability in the dark. Another individual may compensate by using sensory substitution from light touch on a cane or walker, which is helpful in maintaining stability in the dark but may become an obstacle when the person needs to step quickly to the side to recover their equilibrium in response to a perturbation. Thus, quantifying somatosensory loss in the feet, although helpful, cannot fully predict balance function because function also depends on strategies that individuals use to accomplish stability for a particular task given the impairments [10].

Fear of falling has been assessed in two ways. Typically, it is measured by a single questionnaire item. This approach has the advantage of being simple, hence suitable for older people with mild cognitive impairment, and inclusive, as different aspects of fear of falling can be tapped by the same item. However, the single-item methodology is unable to distinguish between excessive fear and rational anticipation of future falls; it makes no distinction between perceived risk of falling and fear of the consequences of falling, and it also leaves unanswered the question of what aspects of falling are anticipated and feared. Consequently, questionnaires have been developed to measure beliefs concerning personal capacity to carry out activities safely without falling (fallrelated self-efficacy). These have been shown to correlate with single-item measures of fear of falling and to predict decline in activities of daily living. Such questionnaires have an exclusively functional focus, as they assess the impact of perceived falling risk on activity-related confidence [11].

Fear of falling was originally conceptualized and measured as a dichotomous parameter (i.e. present versus absent). The simple presence or absence of fear of falling was used in early research studies but the limitation was its inability to determine different degrees of fear of falling which existed across different circumstances and had different impact on physical function [4]. It is difficult to quantify the fear of fall by simply asking the person to rate him or her fear of falling.

Even though a majority of the commonly used questionnaires were initially developed in English language, it is still possible to translate these questionnaires into another language. The MFES has been translated into several languages including Persian, Dutch, Chinese and Swedish, but not into any Indian languages. Not all translations were translated back into English or were devised using a multistep process. Since about $41 \%$ of the Indian population is Hindi speaking, a measure for falls efficacy in Hindi was warranted. About $88 \%$ of the people residing in Gujarat, use Gujarati as their mother tongue, therefore translation of MFES into Gujarati would be useful for people whose first language is Gujarati. Translation of the questionnaire into the mother tongue makes it simpler and more comprehensible for the subjects under study. The population in question can get through the questions more accurately and can answer confidently. Also, the researcher can build concrete faith on his interpretations and the consequent result. Thus, this study is aimed at designing Gujarati and Hindi versions of MFES.

However, one should not simply assume that the validity of the items that are translated from one questionnaire to another remains intact. When translating a questionnaire into another language, proper linguistic as well as cultural validation is necessary Therefore, it is important to translate the questionnaire using a process that incorporates the correct psychometric properties, such as the validity and reliability, before one can declare the translated questionnaire ready to be used. This is due to the fact that validity is content specific and it cannot be translated in a literal manner [12]. Also, the cultural context of the second language, such as the words used, is different from the original language.

It is important to have a simple and validated screening tool to discriminate or predict the potential fallers. The fear of falling is closely related to falls and MFES is validated for measuring the level of fear for elderly fallers in western countries. However, there are no Gujarati and Hindi versions of MFES available and nor are they validated for use in elderly population. Therefore, the aim of present study is to provide translations of MFES into Hindi and Gujarati and to assess the validity of these versions to assess the fall related efficacy [13-23]. 


\section{Annals of Physiotherapy \& Occupational Therapy}

\section{Materials \& Methods}

The study proposal was forwarded to Institutional Ethical Committee for ethical approval and after acquiring approval, the study was initiated.

\section{Translation and Back Translation}

The instrument of the MFES was translated and backtranslated using the technique described by Beaton and Claire [24] after obtaining ethical clearance from Institutional Ethical Committee, the MFES was first translated from English to Hindi and Gujarati by certified bilingual translators. It was then back translated to English by another set of translators, who were blinded to original version. (Gujarati \& Hindi MFES-1). The back translation was matched with the original version and the discrepancies that arose were noted.

\section{Expert Review}

The translated scales were then evaluated and the item equivalence and content relevance were assessed by three expert senior physiotherapists from Sumandeep Vidyapeeth, Vadodara and MGM University, Mumbai. A Likert scale rating was given to each rater, ranging from 1 to 5 (1-very poor, 2-poor, 3-satisfactory, 4-good, 5excellent). If the rating for any item was less than 3 or 3 , it suggested that that item needed a change. These changes were suggested by the raters and corrections were implemented (ANNEXURE III-GUJARATI \& HINDI MFES).

After obtaining final versions, they were evaluated by language professionals, who were Hindi and Gujarati language teachers of English medium secondary school. They were also required to rate each item on the Likert scale. Since no item scored less than 3, the translations were taken as approved.

\section{Pilot Testing}

A pilot testing was performed prior on 10 subjects, wherein each subject completed the questionnaire, and was interviewed what he or she thought about the meaning of each questionnaire item and the chosen response. The 10 subjects of the pilot study were asked to complete the questionnaire and to comment on readability, comprehensiveness, and perceived relevance of the items. Since there were no gross differences between the participant's interpretation and the original meanings, the questionnaire was finalized.

\section{Subject Recruitment}

The subjects were recruited from geriatric homes; social and community gathering places (clubs and parks etc.) in and around Vadodara city with prior official permission. The nature and purpose of the study was explained to the participants. Signed informed consent was obtained from participants.

Upon agreement to participate, the participants were screened to match the inclusion and exclusion criteria. All community dwelling older adults aged 60 years or above of both genders; and who were able to walk with or without assistive devices were included. The subjects, who were unable to walk without the assistance of another person; who were unable to understand and answer the questionnaire; and who were bedbound / confined to home were excluded from the study.

A total of 117 subjects were screened for the study, out of which 7 matched the exclusion criteria. There were 12 dropouts due to various reasons like leaving the institute premises, occurrence of fall between the 2 sessions and bed rest and worsening of general health.

The subjects were asked to complete a health status questionnaire providing information on demographic data and fall history. The subjects were required to complete the Modified Falls Efficacy scale in Hindi/Gujarati on one to one basis. The subjects were given questionnaires in their mother tongue. For those who could not read and answer the questionnaire, the questionnaire was planned to be administered on an interview basis. Any doubts regarding the questionnaire were clarified by the investigator. The average score was obtained by the total score divided by answered items. The possible final score of the MFES ranges from 0to1. It takes approximately 5 minutes to complete and requires no sophisticated equipment, making it useful in clinical settings.

Along with this, balance also was assessed using Berg Balance test and the scores were recorded. The Berg Balance Scale is a performance-based measure designed to monitor performance during balance activities, and to predict multiple falls in community-dwelling and institutionalized older adults. It takes about 15-20 minutes to be carried out. Berg et al contend that scores below 45 indicate that balance is impaired, with an increased risk for falls. Before performing the BBS, each subject was demonstrated the procedure for better understanding.

A total of 98 subjects were recruited in the study, out of which 70 completed the Gujarati MFES and 28 subjects were recruited for Hindi MFES. All the data was documented in data collection forms and the scores were calculated. A Master chart was prepared in MS Excel sheet 


\section{Annals of Physiotherapy \& Occupational Therapy}

and all the collected data was entered into it and used for data analysis.
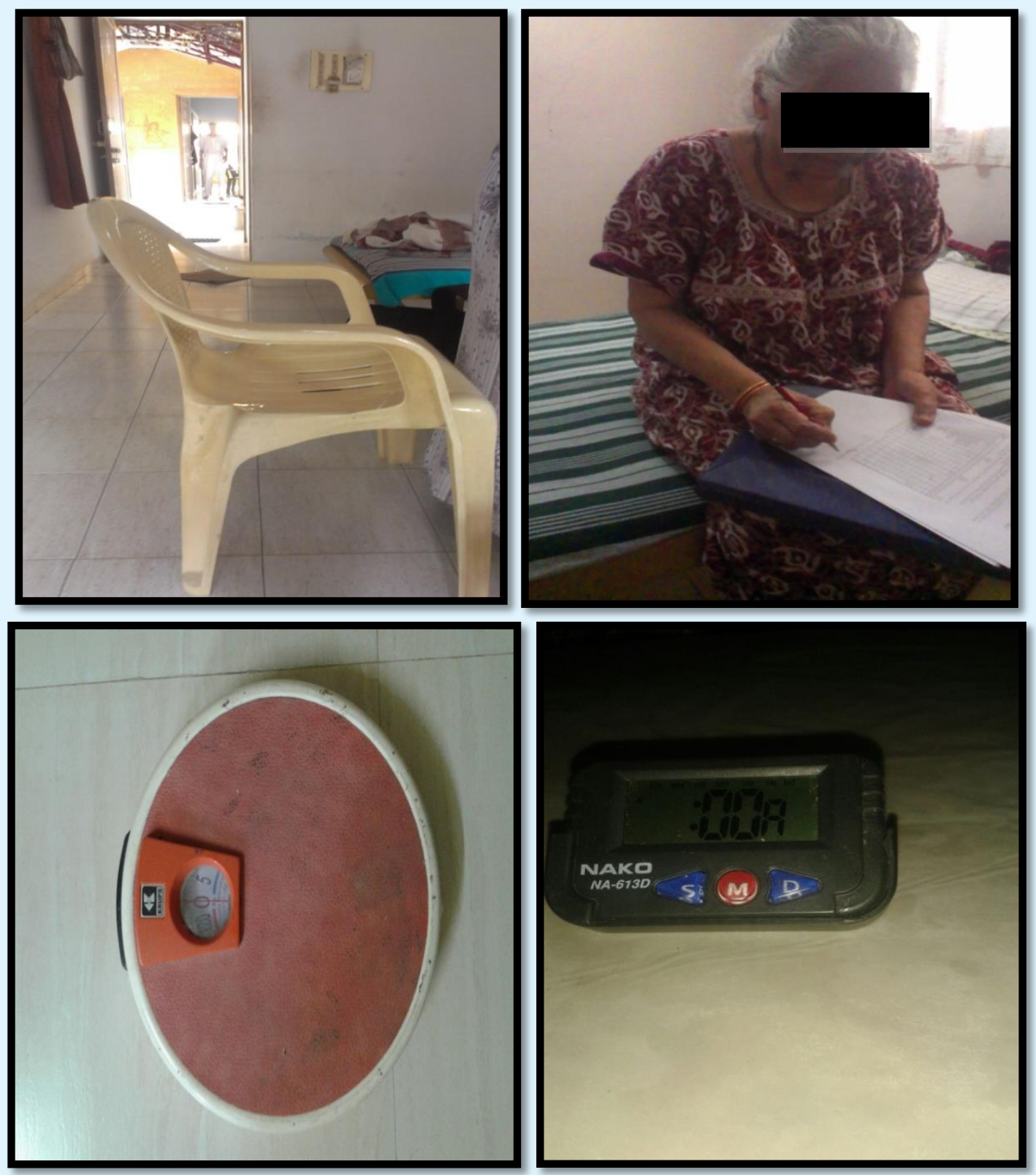

Figure 3: Materials Used. 

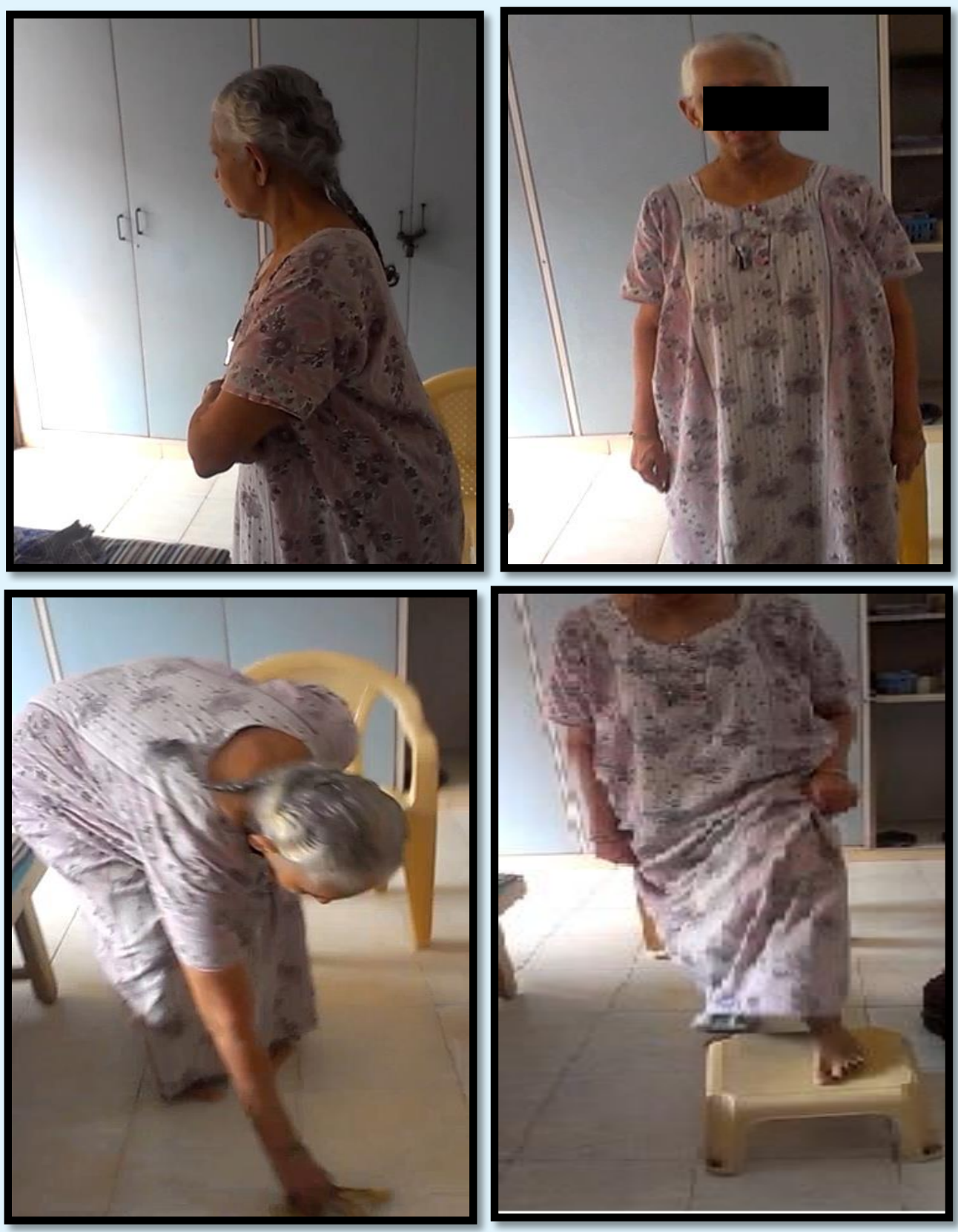

Figure 4: Berg Balance Test.

\section{Statistical Analysis}

a. The statistical analysis was performed using Statistical Package for Social Science Version 16. Descriptive statistics including mean, standard deviation and confidence interval were computed for all variables. Internal consistency for all the items of MFES 1 and MFES 2 was assessed for through Cronbach's alpha coefficient. b. Convergent validity was assessed using the Pearson coefficient from correlation analyses with continuous variable of BBS scores.

c. Content validity of the questionnaire was assessed by expert panel review. The expert panel focused on reviewing and deciding whether the wordings used in the translated questionnaire are appropriate.

d. Correlation coefficient values over .60 were considered as strong correlation; values between .30 and .60 as moderate correlation; and values under .30 as weak 
correlation [25]. Cronbach alpha values over .80 were considered excellent; values over.70, good; and values between .60 and .70, acceptable [26]. P values of $<0.05$ were regarded as statistically significant.

\section{Results}

A total of 70 subjects were recruited for validation of Gujarati version of MFES and 28 for validation of Hindi
MFES. 70 subjects recruited for Gujarati version of MFES. There were $31.4 \%(n=22)$ males and $68.6 \% \quad(n=48)$ females. 28 subjects recruited for Hindi version of MFES, $46.4 \%(n=13)$ participants were males, while $53.6 \%$ $(n=15)$ subjects were females. The mean age of subjects of Gujarati MFES was 75.49 with an SD of 7.495. The mean age of subjects who filled up Hindi MFES was 71.71 with an SD OF 7.473. Descriptive statistics of demographic data shown in Table 1.

\begin{tabular}{|c|c|c|c|c|}
\hline N=70 & Minimum & Maximum & Mean & Std. Deviation \\
\hline Age & 60 & 94 & 75.49 & 7.945 \\
\hline Weight & 38 & 90 & 61.34 & 11.662 \\
\hline BMI & 16.02 & 41.01 & 25.46 & 5.408 \\
\hline
\end{tabular}

Table 1A: Gujarati version of MFES.

\begin{tabular}{|c|c|c|c|c|}
\hline N=28 & Minimum & Maximum & Mean & Std. Deviation \\
\hline AGE & 60 & 88 & 71.71 & 7.473 \\
\hline WEIGHT & 45 & 86 & 67.14 & 9.364 \\
\hline BMI & 18.34 & 38.08 & 27.015 & 4.37 \\
\hline
\end{tabular}

Table 1B: Hindi version of MFES.

\begin{tabular}{|c|c|c|c|c|}
\hline & \multicolumn{2}{|c|}{ Gujarati MFES } & \multicolumn{2}{c|}{ Hindi MFES } \\
\hline & Mean & STANDARD Deviation & Mean & STANDARD Deviation \\
\hline MFES & 0.781 & 1.899 & 0.861 & 2.05 \\
\hline BBS & 43.67 & 3.54 & 46.39 & 3.372 \\
\hline
\end{tabular}

Table 2: Mean Values of Berg Balance Scale Scores and Modified Falls Efficacy Scale Scores

\begin{tabular}{|c|c|c|c|c|}
\hline \multicolumn{2}{|c|}{ Gujarati MFES } & & \multicolumn{2}{c|}{ Hindi MFES } \\
\cline { 4 - 5 } & Cronbach's & \multirow{2}{*}{ ICC } & \multirow{2}{*}{ ICC } \\
\cline { 4 - 5 } & Alpha & & Cronbach's & 1 \\
\hline ITEM 1 & 0.997 & 0.993 & 1 & 0.836 \\
\hline ITEM 2 & 0.989 & 0.979 & 0.911 & 0.947 \\
\hline ITEM 3 & 0.963 & 0.929 & 0.973 & 0.883 \\
\hline ITEM 4 & 0.874 & 0.775 & 0.936 & 0.778 \\
\hline ITEM 5 & 0.945 & 0.895 & 0.875 & 0.456 \\
\hline ITEM 6 & 0.989 & 0.979 & 1 & 0.633 \\
\hline ITEM 7 & 0.964 & 0.93 & 0.627 & 0.813 \\
\hline ITEM 8 & 0.972 & 0.945 & 0.775 & 0.696 \\
\hline ITEM 9 & 0.973 & 0.948 & 0.897 & 0.69 \\
\hline ITEM 10 & 0.921 & 0.854 & 0.821 & 0.78 \\
\hline ITEM 11 & 0.873 & 0.774 & 0.817 & 0.89 \\
\hline ITEM 12 & 0.901 & 0.819 & 0.876 & 0.987 \\
\hline ITEM 13 & 0.983 & 0.967 & 0.981 & 0.942 \\
\hline ITEM 14 & 0.914 & 0.842 & 0.993 & \\
\hline TOTAL & 0.992 & 0.984 & & 0.89 \\
\hline
\end{tabular}

Table 3: Cronbach's Alpha and Intraclass Correlation Coefficient (ICC) Values of MFES Items. 


\section{Annals of Physiotherapy \& Occupational Therapy}

\section{Concurrent Validity Analysis}

\section{Correlation between MFES and BBS}

\begin{tabular}{|c|c|}
\hline \multicolumn{2}{|c|}{ MFES-1 } \\
\hline Pearson correlation coefficient (r) & 0.799 \\
\hline P-value & 0 \\
\hline
\end{tabular}

Table 4: Correlation between Gujarati Version of MFES and BBS.

\begin{tabular}{|c|c|}
\hline \multicolumn{2}{|c|}{ MFES-1 } \\
\hline Pearson correlation coefficient & 0.522 \\
\hline P-value & 0.004 \\
\hline
\end{tabular}

Table 5: Correlation between Hindi Version of MFES and BBS.

The Gujarati MFES scores showed a strong positive correlation with Berg Balance Scale scores; while the Hindi MFES scores showed a moderate positive correlation with Berg Balance scale. The higher MFES scores were significantly associated with greater balance performance. (Gujarati MFES: $\mathrm{r}=0.820, \mathrm{P}$ value $=0.000$; Hindi MFES: $r=0.565$, $P$ value $=0.002$ ) [27].

\section{Correlation of MFES and BBS with Age}

\begin{tabular}{|c|c|c|}
\hline & Gujarati MFES & Hindi MFES \\
\hline $\begin{array}{c}\text { Pearson correlation } \\
\text { coefficient }\end{array}$ & -0.36 & -0.302 \\
\hline P-value & 0.002 & 0.118 \\
\hline
\end{tabular}

Table 6: Correlation between MFES and age.

The MFES scores showed a negative correlation with age, stating that with an increase in age, the fall-related efficacy reduces. (Gujarati MFES: $r=-0.351$, $P$ value $=0.003$; Hindi MFES: $r=-0.297, P$ value $=0.125$ ) [28]

\section{Convergent Validity}

\begin{tabular}{|c|c|c|}
\hline & Gujarati MFES & Hindi MFES \\
\hline $\begin{array}{c}\text { Pearson correlation } \\
\text { coefficient }\end{array}$ & 0.72 & 0.56 \\
\hline P-value & 0.003 & 0.12 \\
\hline
\end{tabular}

Table 7: Correlation between MFES and fear of falling.

The MFES scores showed a positive correlation with fear of falls, suggesting that individuals with fear of falling had significantly lower values of MFES [29].

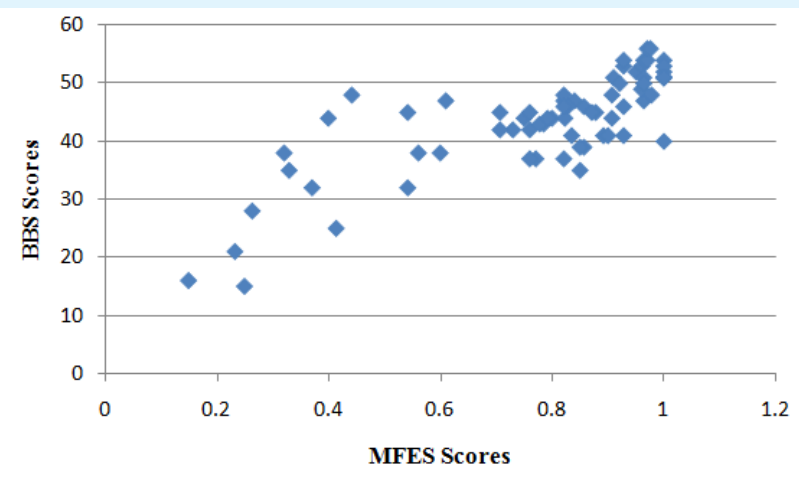

Figure 5: Scatter plot of MFES versus BBS scores.

\section{Discussion}

The English version of Modified Falls Efficacy scale is a self-report measure of falls efficacy, also commonly known as fear of falling. It is a modification of original 10 item Falls Efficacy Scale. The English version of MFES has high test-retest reliability and internal consistency (ICC = 0.93 and Cronbach's alpha $=0.95$ ). The MFES appears to be a reliable and valid measure of falls self-efficacy. It may be a useful addition in the comprehensive assessment of older people with balance disturbance at falls. Apart from MFES, other scales are also available to assess the fall related efficacy. The ABC scale also has excellent psychometric properties with high reliability and validity. However, some items in the $\mathrm{ABC}$ scale are not suitable for India such as icy sidewalk. The items on the original FES refer exclusively to very basic activities of daily living that only frail or disabled people would be likely to have difficulty with, and do not include the more demanding activities which may be the principal cause for concern among higher functioning older people. They don't take into account various outdoor activities which require greater confidence. So MFES was chosen for translation into Hindi and Gujarati. Thus the goal of this study was to evaluate the validity and reliability of Gujarati and Hindi versions of MFES [30].

This study shows that, among the older adults living in community or institutionalized elderly, this adapted Gujarati version of the MFES has excellent validity. In particular, results confirmed the hypotheses of significant association with measures of balance.

\section{Concurrent Validity}

The result of this study showed the association between the falls- related efficacy and balance performance in the elderly people. The Gujarati MFES 
scores showed a strong positive correlation with Berg Balance Scale scores; while the Hindi MFES showed a moderate positive correlation with the BBS; higher MFES scores were associated with greater balance confidence. (Gujarati MFES: $r=0.820, \mathrm{P}$ value $=0.000$; Hindi MFES: $\mathrm{r}=0.565$, $\mathrm{P}$ value=0.002). It showed the relationship between the falls efficacy and the balance ability during the functional tasks. This relation between the fear of falling and the balance was in agreement with Maki et al 's results which showed that older adults who reported a fear of falling demonstrated larger amplitude of postural sway when blindfolded and poorer scores when timed on a one-leg stance test compared to those who did not report fear of falling [31].

The analysis of the means of individual items of Hindi and Gujarati MFES reveals that the subjects had maximum falls efficacy in component 12 "Light gardening or hanging out the washing". And the subjects showed minimum efficacy, i.e. greatest fear of fall in item 13 "Crossing roads" [32].

During the translation phase of the study, after the first translation, the item 5 'Get in/out of the bed' was translated to Gujarati as પથારીમાંસૂવુંપથારીમાંથીબહારઆવવું. On back translation, it was found to be 'sleeping in the bed/ getting out up from the bed', which had a discrepancy with the original meaning. Also the item 8, 'Reach into cabinets/coset' was translated as કબાટોકેખાનાંસુધીપહોંચવુ, which had a discrepancy with the original meaning. So these discrepancies were sorted by the expert panel and were reframed as attached in the annexure. For Hindi version of MFES, no major changes were suggested. The language professionals, who were Hindi and Gujarati language teachers of English medium secondary school, rated each item on the Likert scale above 3(good/excellent) so no changes were required. Then a pilot study was carried out with 10 subjects, who were asked to elaborate their understanding of each of the component, and the responses were recorded. Since their opinion was unanimous, the Gujarati and Hindi versions were finalized [33].

Apart from the items in MFES, the subjects were asked to describe about some other likelihood events that gave rise to fear of falls in them. About 30\% of subjects described various events which were not mentioned in MFES but gave rise to fear of fall like walking on wet floor, walking on uneven surfaces, slope walking, driving vehicle, going to crowded places, etc. These components were not statistically analyzed but maximum responses were about walking on wet floor, walking on uneven surfaces, going to crowded places.
The Hindi version of Modified Falls efficacy scale did not show a strong correlation with Berg Balance Scale( $r=0.565$, $\mathrm{P}$ value $<0.001)$, nor did it show significant negative correlation with age (Hindi MFES: $r=-0.297, P$ value $=0.125)$. The probable reasons for these findings may be less number of subjects $(n=28)$ to establish validity, and a dearth of elderly population in Vadodara who have Hindi as their first language. Thus, further research with a larger sample size for Hindi MFES may be required for further validation [34].

\section{Conclusion}

The Gujarati version of MFES is a valid measure to estimate fall-related efficacy in older adults who have Gujarati as their first language. (Cronbach's alpha-0.993; $r=0.7)$. It is adequate from a semantic and linguistic point of view and is applicable to community-dwelling elderly Indian population. The Hindi version of MFES can have its validity established with a larger sample size than the present study. (Cronbach's alpha - 0.992; r=0.5).

\section{References}

1. Dsouza SA, Rajashekar B, Dsouza HS, Kumar KB (2014) Falls in Indian older adults: a barrier to active ageing. Asian J Gerontol Geriatr 9: 33-40.

2. Suraj Kumar, Venu Vendhan G (2008) Relationship Between Fear of Falling, Balance Impairment and Functional Mobility in Community Dwelling Elderly. IJPMR 19(2): 48-52.

3. Falls in older people: risk factors and strategies for prevention. Inj Prve 9(1).

4. LuiWai Man (2005) Validity of the Chinese version of Modified Falls Efficacy Scale in predicting falls among community-dwelling elderly in Hong Kong.

5. Hartholt KA, van Beeck EF, Pollinder S, van der Velde N, van Lieshotu EM, et al. (2011) Societal consequences of falls in the older population: injuries, healthcare costs, and long-term reduced quality of life. J Trauma 71(3): 748-753.

6. Mendes de Leon CF, Seeman TE, Baker DI, Richardson ED, Tinetti ME (1996) Self efficacy, physical decline, and change in functioning in community-living elders: a prospective study. J Gerontol B Psychol Sci Soc Sci 51: S183-S190.

7. Zijlstra GA, van Haastregt JC, van Rossum E, van Eijk JT, Yardley L, et al. (2007) Interventions to reduce fear of falling in community-living older people: a 


\section{Annals of Physiotherapy \& Occupational Therapy}

systematic review. Journal of the American Geriatrics Society 55(4): 603-615.

8. Vellas BJ, Wayne SJ, Romero LJ, Baumgartner RN, Garry PJ (1997) Fear of falling and restriction of mobility in elderly fallers. Age Ageing 26(3): 189-193.

9. Hill KD, Schwarz JA, Kalogeropoulos AJ, Gibson SJ (1996) Fear of falling revisited. Arch Phys Med Rehabil 77(10): 1025-1029.

10. Horak FB (2006) Postural orientation and equilibrium: what do we need to know about neural control of balance to prevent falls?. Age and Ageing 35(2): ii7-ii11.

11. Yardley L, Smith H (2002) A Prospective Study of the Relationship between Feared Consequences of Falling and Avoidance of Activity in Community-Living Older People. Gerontologist 42(1): 17-23.

12. Grylka- Baeschlin S, van Teijlingen E, Stoll K, Gross MM (2015) Translation and validation of the German version of the Mother-Generated Index and its application during the postnatal period. Midwifery 31(1): 47-53.

13. Homann B, Plaschg A, Grundner M, Haubenhofer A, Griedl T, et al. (2013) The impact of neurological disorders on the risk for falls in the community dwelling elderly: a case-controlled study. BMJ Open 3(11): e003367.

14. Boulgarides LK, McGinty SM, Willett JA, Barnes CW (2003) Use of clinical based impairments to Predict Falls by Community-Dwelling Older Adults. Phys Ther 83(4): 328-339.

15. Muir SW, Gopaul K, Montero Odasso MM (2012) The role of cognitive impairment in fall risk among older adults: a systematic review and meta-analysis. Age Ageing 41(3): 299-308.

16. Hatch J, Gill-Body KM, Portney LG (2003) Determinants of Balance Confidence in CommunityDwelling Elderly People. Phys Ther 83(12): 10721079.

17. Barak Y, Wagenaar RC, Holt KG (2006) Gait Characteristics of Elderly People With a History of Falls: A Dynamic Approach. Phys Ther 86(11): 15011510.

18. Studenski, Newman AB, Talkowski JB, Brach JS, Stephanie (2008) Walking Activity in Older Adults: History, Balance Performance, and Gait Speed on
Impact of Health Perception, Balance Perception, Fall. Phys Ther 88: 1474-1481.

19. Schepens S, Sen A, Painter JA, Murphy SL (2012) Relationship Between Fall-Related Efficacy and Activity Engagement inCommunity-Dwelling Older Adults: A Meta-Analytic Review. Am J Occup Ther 66(2): 137-148.

20. Belgen B, Beninato $M$, Sullivan PE, Narielwalla K (2006) The Association of Balance Capacity and Falls Self-Efficacy With History of Falling in CommunityDwelling People With Chronic Stroke. Arch Phys Med Rehabil 87(4): 554-561.

21. Conradsson M, Lundin-Olsson L, Lindelöf N, Littbrand H, Malmqvist L, et al. (2007) Berg Balance Scale: Intrarater Test-Retest Reliability among Older People Dependent in Activities of Daily Living and Living in Residential Care Facilities. Phys Ther 87(9): 11551163.

22. Muir SW, Berg K, Chesworth B, Speechley M (2008) Use of the Berg Balance Scale for Predicting Multiple Falls in Community- Dwelling Elderly People: A Prospective Study. Physical Therapy 88(4): 449-459.

23. Hill KD, Schwarz JA, Kalogeropoulos AJ, Gibson SJ (1996) Fear of falling revisited. Arch Phys Med Rehabil 77(10): 1025-1029.

24. Beaton DE, Bombardier C, Guillemin F, Ferraz MB (2000) Guidelines for the Process of Cross-Cultural Adaptation of Self-Report Measures. SPINE 25(24): 3186-3191.

25. Waltz CF, Strickland OL, Lenz ER (2010) Measurement in Nursing and Health Research. Springer Publishing Co, New York.

26. Cronbach LJ, Meehl PE, Minneapolis MN Construct Validity in Psychological Tests. Cronbach LJ, Meehl PE Minneapolis.

27. Edwards N, Lockett D (2008) Development and validation of a modified falls-efficacy scale. Disability and Rehabilitation 3(4): 193-200.

28. Mosallanezhad Z, Salavati M, Hellström K, Reza Sotoudeh G, Nilsson Wikmar L, et al. (2011) Crosscultural adaptation, reliability and validity of the Persian version of the modified falls efficacy scale 33(25-26): 2446-2453.

29. Ulus Y, Durmus D, Akyol Y, Terzi Y, Bilgici A, et al. (2012) Reliability and validity of the Turkish version 


\section{Annals of Physiotherapy \& Occupational Therapy}

of the Falls Efficacy Scale International (FES-I) in community-dwelling older persons. Arch Gerontol Geriatr 54(3): 429-433.

30. Powell LE, Myers AM (1995) The Activities-specific Balance Confidence (ABC) Scale. J Gerontol A Biol Sci Med Sci 50(1): 28-34.

31. Bishop MD, Patterson TS, Romero S, Light KE (2010) Improved Fall-Related Efficacy in Older Adults Related to Changes in Dynamic Gait Ability. Phys Ther 90(11): 1598-1606.
32. Average Indian's life expectancy up 4.6 year: Kounteya Sinha, TNN | Oct 2, 2012, 01.58AM IST.

33. Barazini F, Diurni M, Ceccon F, Poloni N, Cazzamalli S, et al. (2009) Fall related injuries in nursing home setting: is poly pharmacy a risk factor?. BMC Health Services Research 9: 228.

34. O'Halloran AM, Pénard N, Galli A, Fan CW, Robertson IH, et al. (2011) Falls and falls efficacy: the role of sustained attention in older adults. BMC Geriatr 11: 85. 